\title{
Comparing robotic, laparoscopic and open cystectomy: A systematic review and meta-analysis
}

\author{
Thomas Fonseka ${ }^{1}$, Kamran Ahmed ${ }^{2}$, Saied Froghi ${ }^{2}$, Shahid A Khan ${ }^{3}$, \\ Prokar Dasgupta ${ }^{2}$, Mohammad Shamim Khan ${ }^{2}$ \\ ${ }^{1}$ King's College London School of Medicine, London, UK; \\ ${ }^{2}$ MRC Centre for Transplantation, King's College London, King's Health Partners, Department of Urology, Guy's Hospital, London, UK; \\ ${ }^{3}$ Surrey and Sussex Healthcare NHS Hospital, Redhill, UK.
}

\begin{abstract}
Summary Objective: To conduct a systematic review and meta-analysis comparing outcomes between Open Radical Cystectomy (ORC), Laparoscopic Radical Cystectomy (LRC) and Robot-assisted Radical Cystectomy (RARC). RARC is to be compared to LRC and ORC and LRC compared to ORC.

Material and methods: A systematic review of the literature was conducted, collating studies comparing RARC, LRC and ORC. Surgical and oncological outcome data were extracted and a meta-analysis was performed.

Results: Twenty-four studies were selected with total of 2,104 cases analyzed. RARC had a longer operative time (OPT) compared to LRC with no statistical difference between length of stay (LOS) and estimated blood loss (EBL). RARC had a significantly shorter LOS, reduced EBL, lower complication rate and longer OPT compared to ORC. There were no significant differences regarding lymph node yield (LNY) and positive surgical margins (PSM.) LRC had a reduced EBL, shorter LOS and increased OPT compared to ORC. There was no significant difference regarding LNY.

Conclusion: RARC is comparable to LRC with better surgical results than ORC. LRC has better surgical outcomes than ORC. With the unique technological features of the robotic surgical system and increasing trend of intra-corporeal reconstruction it is likely that RARC will become the surgical option of choice.
\end{abstract}

KEY WORDS: Radical cystectomy; Robotic assisted radical cystectomy; Laparoscopic radical cystectomy; Open radical cystectomy; Bladder cancer.

Submitted 22 December 2014; Accepted 31 December 2014

\section{INTRODUCTION}

Open Radical Cystectomy (ORC) is currently regarded as the gold standard surgical treatment for muscle-invasive and non-muscle invasive high-grade recurrent bladder cancer (1). However, the procedure is associated with high morbidity including increased blood loss, prolonged hospital stay and slower recovery.

Laparoscopic Radical Cystectomy (LRC) was developed around two decades but Robot-assisted Radical Cystecto- my (RARC) has since emerged as a viable alternative. RARC has been reported to be associated with fewer complications compared to LRC (2) and the appeal of the robotic surgical system has led to the adoption of the procedure worldwide. However the production of high-quality evidence that measures the relative merits of ORC, LRC and RARC is lacking with surgeons having to rely on systematic review. This article aims to compare surgical and oncological outcomes of ORC, LRC and RARC.

\section{Methods}

\section{Eligibility criteria}

Data were collected on all patients over the age of 60 with muscle-invasive bladder cancer undergoing RARC, LRC or ORC. Surgical outcomes were; operative time (OPT), estimated blood loss (EBL), length of stay (LOS) and complication rate 90 days post-operatively. Oncological outcomes were; lymph node yield (LNY) and positive surgical margins (PSM). Comparisons were made between RARC, LRC and ORC and the outcomes of interest measured included both surgical and oncological outcomes.

The studies forming the current meta-analysis include comparative studies, either retrospective or prospective, as well as randomized control trials.

For a study to be included in our analysis it had to fulfill the following criteria. The study had to:

- Compare outcome measures of two or all three surgical techniques (ORC, LRC and RARC).

- Use quantitative data for at least one outcome measure.

- Be a high quality study.

If it was one of two studies that were produced by the same institution, it was ensured the data were mutually exclusive.

Studies were excluded if they:

- Lacked reporting of the desired outcome measures listed above or presented the data in such a way that it was not possible to carry out an analysis for the study.

- Reported on only one of the techniques of ORC, LRC and RARC.

- Were written in non-English language. 


\section{Information sources}

A systematic review of the literature was conducted using the following databases; PubMed, Medline, the Cochrane Library and EMBASE. The reference lists of reviews were also cross-referenced. The last search was conducted on $11 / 12 / 2014$

\section{Search}

The following search terms were used: "Open cystectomy", "Open radical cystectomy", "Laparoscopic cystectomy", "Laparoscopic assisted cystectomy", "Laparoscopic radical cystectomy", "Laparoscopic assisted radical cystectomy", "Robotic cystectomy", "Robot* assisted cystectomy", "Robotic radical cystectomy", "Robot* assisted radical cystectomy", "Robot* assisted laparoscopic cystectomy", "Robot* assisted laparoscopic radical cystectomy", "Minimally invasive", "Bladder cancer". The search terms were combined to ensure as many studies as possible that compared ORC, LRC and RARC, or any combination were included.

\section{Study selection}

Studies were selected by two reviewers (T.F and S.F), independently. Where the decision was split and agreement could not be made, the study was included so as to include as many studies as possible.

\section{Data items}

After selection of the studies, the following data were extracted; primary author of the study, year of publication, country of study, study design, study exclusion criteria (if mentioned), total number of patients undergoing ORC, LRC and RARC, study population characteristics (mean age, mean BMI, mean American Society of Anesthesiologists (ASA) grade, Charlson's Co-morbidity Index, gender, and pathological stage). For each technique (ORC, LRC and RARC), the following data were recorded: primary author of the study, year of publication, total number of patients undergoing each urinary diversion type (conduit or neo-bladder), total number of patients requiring blood transfusion, surgical outcomes (as previously listed) and oncological outcomes (as previously listed). Complications were assessed using the Clavien-Dindo grading system (3).

\section{Risk of bias in individual studies}

The Newcastle-Ottawa Quality Assessment Scale (4) was used to assess the quality of the studies. It was tailored to suit the analysis of the studies included in this evaluation. Areas analyzed for quality were patient selection, including representativeness of the exposed cohort, comparability of cohorts, and assessment of outcomes. Studies which were rated with five or more stars were deemed to be high-quality. The entire analysis was conducted using Review Manager Version 5 (The Cochrane Collaboration, Software Update, Oxford).

\section{Summary measures}

To assess whether there was a statistically significant difference between data of a dichotomous nature, the odds ratio (OR) was calculated. The OR is a measure of the probability of an event occurring in an RARC patient group compared to either ORC or LRC patient groups or
LRC compared to ORC. When comparing adverse events, where an OR value was less than one it implied that RARC was favored. In order for the point estimate of the OR to be considered statistically significant at the $\mathrm{P}<$ 0.05 level, the $95 \%$ CI must not have included the value of one. When handling continuous data the mean weighted difference (MWD) was used instead of the OR. A negative MWD value indicated RARC was favored.

\section{Synthesis of results}

Guidance was sought from the Cochrane Collaboration as well as information from the QUORUM guidelines (5) to provide the framework of the statistical analysis.

When studies reported of medians, ranges or confidence intervals for continuous variables, statistical algorithms were used to derive the appropriate means and standard deviations. The OR for continuous variables could be calculated using the Mantle-Haenszel Chi square method with the 'random effects' meta-analytical technique. The 'random effects' model is particularly useful when conducting surgical research. This is because it takes into account the almost inevitable natural variation inherent between studies. Subsequently a more conservative OR is produced. For both OR and MWD, corresponding 95\% CI's were calculated. Regarding the Forest plots produced, a square represents the point estimate of the treatment effect, that is the OR or MWD, with a horizontal bar going through the square showing the $95 \% \mathrm{CI}$. The summary measure of the pooled studies with $95 \%$ Cl's is represented by a diamond.

\section{RESULTS}

\section{Study selection}

The initial literature search identified 598 papers, which matched the search criteria. Of these, 486 papers were eliminated due to broad incoherency with the aims of this study. Of the 112 remaining, a further 83 papers were excluded based on the exclusion criteria as outlined above. On more in depth examination of the 29 remain-

\section{Figure 1.}

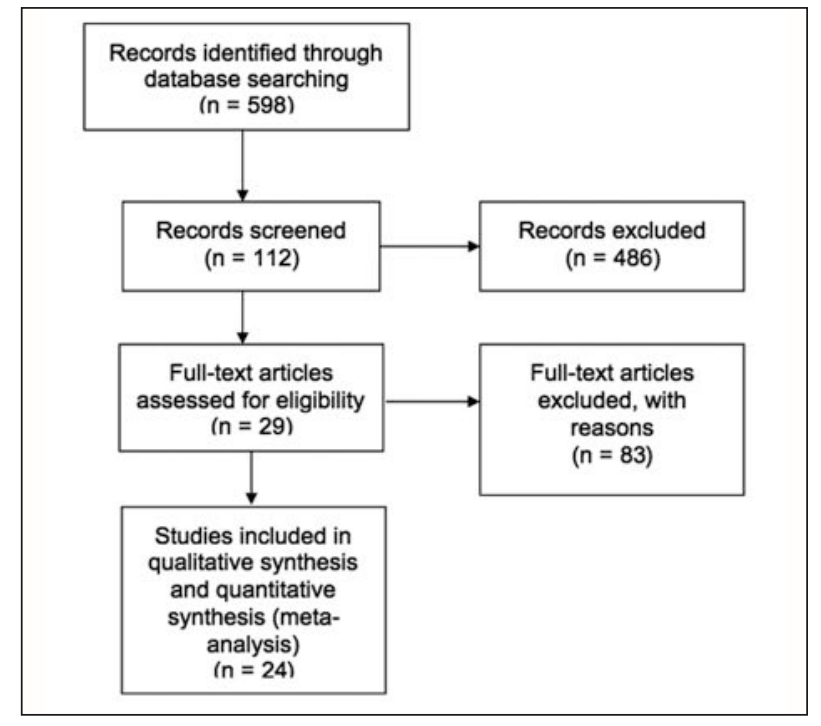


Table 1.

Study characteristics. Matching: 1 - Age; 2 - BMI; 3 - ASA; 4 - Charlson; 5 - Gender; 6 - Pathological stage; 7 - Urinary diversion type. Study type: RCT - Randomized control trial; R - Retrospective, P - Prospective.

\begin{tabular}{|c|c|c|c|c|c|c|c|c|c|c|c|}
\hline \multirow[t]{2}{*}{ Study } & \multirow[t]{2}{*}{ Study type } & \multicolumn{3}{|c|}{ Cases } & \multirow[t]{2}{*}{ Matching } & \multicolumn{3}{|c|}{ Mean age, yaers } & \multirow[t]{2}{*}{ Exclusion criteria } & \multicolumn{2}{|c|}{ Study quality Country } \\
\hline & & ORC & LRC & RARC & & ORC & LRC & RARC & & & \\
\hline Abaza 2012 & $\mathrm{R}$ & 120 & NR & 35 & $1,2,6$ & 69.8 & NR & 67.3 & $\begin{array}{l}\text { Undergoing lesser } \\
\text { node dissection due } \\
\text { to a history of radiation, } \\
\text { aortoiliac grafting or } \\
\text { significant comorbidity }\end{array}$ & $* * *$ & USA \\
\hline Abraham 2007 & $\mathrm{P}$ & NR & 20 & 14 & $1,2,3,5,7$ & NR & 77.6 & 76.5 & NR & $* * * *$ & USA \\
\hline Galich 2006 & $P$ & 24 & NR & 13 & $1,2,3,6$ & 63.03 & NR & 66.2 & $\begin{array}{l}\text { Morbid obesity (generally } \\
\text { body mass index_35), } \\
\text { prior pelvic radiation, } \\
\text { or significant medical } \\
\text { comorbidities including } \\
\text { pulmonary obstructive } \\
\text { airway disease }\end{array}$ & $* * * *$ & USA \\
\hline Gondo 2012 & $P$ & 15 & NR & 11 & $1,2,4,5,6,7$ & 69.7 & NR & 68.9 & NR & $* * * *$ & Japan \\
\hline Ha 2010 & $\mathrm{R}$ & 34 & 36 & & $1,2,5,6$ & $55.9(9.8)$ & $67.5(8.9)$ & & & $* * * * *$ & Korea \\
\hline Haber 2008 & $\mathrm{R}$ & 50 & 50 & & 1,2 & 67 & 66 & & & $* * *$ & USA \\
\hline Kader 2013 & $\mathrm{R}$ & 100 & NR & 103 & $1,2,3,5,6,7$ & 66 & NR & 67 & NR & $* * * * *$ & USA \\
\hline Khan 2012 & $\mathrm{P}$ & 52 & 58 & 48 & $1,2,3,5,6,7$ & 65 & 69.8 & 66.5 & NR & $* * * *$ & UK \\
\hline Knox 2013 & $\mathrm{R}$ & 84 & NR & 58 & $1,2,3,5,6,7$ & $67.07(1.2)$ & NR & $65.9(1.2)$ & NR & $* * * *$ & USA \\
\hline Lin 2014 & RCT & 35 & 35 & & $1,2,3,5,6$ & $63.6(8.9)$ & $63.2(9.1)$ & & & $* * * * * * *$ & China \\
\hline Martin 2010 & $\mathrm{P}$ & 14 & NR & 19 & $1,2,3,5,6$ & 68 & NR & 74 & NR & $* * * *$ & USA \\
\hline Musch 2014 & $\mathrm{P}$ & 42 & & 100 & $1,2,3,4,5,6,7$ & $69.0(11.5)$ & & $71.4(9.4)$ & & $* * * * * * *$ & Germany \\
\hline Nepple 2013 & $R$ & 29 & NR & 36 & $1,2,5,6,7$ & 67 & NR & 72 & $\begin{array}{l}\text { Contraindication } \\
\text { to robotic surgery }\end{array}$ & $* * * * *$ & USA \\
\hline Ng 2010 & $P$ & 104 & NR & 83 & $1,2,3,5,6,7$ & 67.2 & NR & 70.9 & NR & $* * * * *$ & USA \\
\hline Nix 2010 & RCT & 20 & NR & 21 & $1,2,3,5,6,7$ & 69.2 & NR & 67.4 & $\begin{array}{l}\text { 1) not surgical candidates, } \\
\text { 2) not allowing randomization } \\
\text { 3) those with preference } \\
\text { for specific surgical modality }\end{array}$ & n & USA \\
\hline Parekh 2013 & RCT & 19 & NR & 20 & $1,2,3,5,6$ & 69.5 & NR & 69.5 & $\begin{array}{l}\text { 1) Inability to give informed } \\
\text { consent, 2) unsafe for } \\
\text { robotic approach, 3) clinical } \\
\text { T4 bladder cancer, 4) clinical } \\
\text { lymph node positive bladder } \\
\text { cancer with grossly enlarged } \\
\text { pelvic or retroperitoneal } \\
\text { lymph nodes, 5) age younger } \\
\text { than } 30 \text { or older than } 90 \\
\text { years and 6) pregnancy. }\end{array}$ & r**** & USA \\
\hline Porpiglia 2007 & $\mathrm{P}$ & 22 & 20 & NR & $1,2,3,5,6,7$ & 71 & 63.5 & NR & NR & $* * * * *$ & Italy \\
\hline Pruthi 2007 & $\mathrm{R}$ & 24 & NR & 20 & $1,6,7$ & 68.2 & NR & 62.3 & NR & $* * *$ & USA \\
\hline Rhee 2006 & $\mathrm{P}$ & 23 & & 7 & $1,2,3,5,6$ & $67(13)$ & & $60(9)$ & & $* * * * * * *$ & USA \\
\hline Richards 2010 & $\mathrm{R}$ & 35 & NR & 35 & $1,2,3,5,6,7$ & 66 & NR & 65 & NR & $* * * *$ & USA \\
\hline Styn 2012 & $\mathrm{P}$ & 100 & NR & 50 & $1,2,3,4,5,6,7$ & 65.6 & NR & 66.6 & NR & $* * * * *$ & USA \\
\hline Sung 2012 & $R$ & 104 & NR & 35 & $1,2,3,5,6$ & 65.9 & NR & 62.2 & $\begin{array}{l}\text { Had undergone radiotherapy } \\
\text { before operation or for } \\
\text { whom palliative treatment } \\
\text { was the primary aim }\end{array}$ & $* * * *$ & Korea \\
\hline
\end{tabular}

ing papers, a subsequent 5 were removed for not meeting the requirements of the inclusion criteria. Thus 24 studies were included in the final quantitative and qualitative analysis (Figure 1) (6-29).

\section{Study characteristics}

Characteristics of all 24 studies included in the analysis are summarized in Table 1 (6-29). There were three randomized control trials with the remaining 21 made up of retrospective and prospective studies. Each study was either 2-arm or 3-arm. For the purposes of the statistics RARC was always regarded as experimental. LRC was also considered experimental except when being compared to RARC, in which case it was used as control. All of the stud- ies included were fairly recent with the oldest published in 2006. The two reviewers who selected the studies were in complete agreement (100\%) about data extraction.

A total of 2,104 cases were analyzed, with 1,100 (52.3\%) undergoing ORC, 276 (13.1\%) LRC and 728 (34.6\%) RARC. Approximately $65 \%$ of patients were male. With regards to the pathological stage of the tumors, the average percentage of tumors that were non-organ confined (pT34) was similar in both RARC and ORC groups. On average $30.1 \%$ of patients reported in the RARC studies had nonorgan confined tumors compared with $29.5 \%$ in ORC group and $14.5 \%$ in the LRC group. On average $28 \%$ of patients undergoing RARC developed complications 90 days post-operatively. Major complications were defined as 
Figure 2.

\begin{tabular}{|c|c|c|c|c|c|c|c|c|c|}
\hline Study or Subgroup & Mean & $\begin{array}{r}\text { ARC } \\
\text { SD }\end{array}$ & Total & Mean & $\begin{array}{l}\text { LRC } \\
\text { SD }\end{array}$ & Total & Weight & $\begin{array}{l}\text { Mean Difference } \\
\text { IV, Random, 95\% CI }\end{array}$ & $\begin{array}{c}\text { Mean Difference } \\
\text { IV, Random, 95\% Ca }\end{array}$ \\
\hline Abraham 2007 & 410 & 25.8 & 14 & 419 & 34.7 & 20 & $31.9 \%$ & $-9.00[-29.34,11.34]$ & -7 \\
\hline Can 2013 & 377.8 & 21.6 & 20 & 300 & 15 & 19 & $33.9 \%$ & $77.80[66.18,89.42]$ & $=$ \\
\hline Khan 2012 & 386.4 & 22.7 & 48 & 316 & 23.3 & 58 & $34.3 \%$ & $70.40[61.61,79.19]$ & $=$ \\
\hline \multirow{2}{*}{\multicolumn{9}{|c|}{$\begin{array}{l}\text { Heterogeneity: Tau }{ }^{2}=1121.69 ; C h i^{2}=56.90 \text {, df }=2(P<0.00001) ; P^{2}=96 \% \\
\text { Test for overall effect: } Z=2.41(P=0.02)\end{array}$}} & \\
\hline & & & & & & & & & $\begin{array}{llll}-200 & -1 & 1 & 1 \\
\text { Favours [RARC] } & 0 & 100 & 200 \\
\text { Favours [LRC] }\end{array}$ \\
\hline
\end{tabular}

Figure 3.

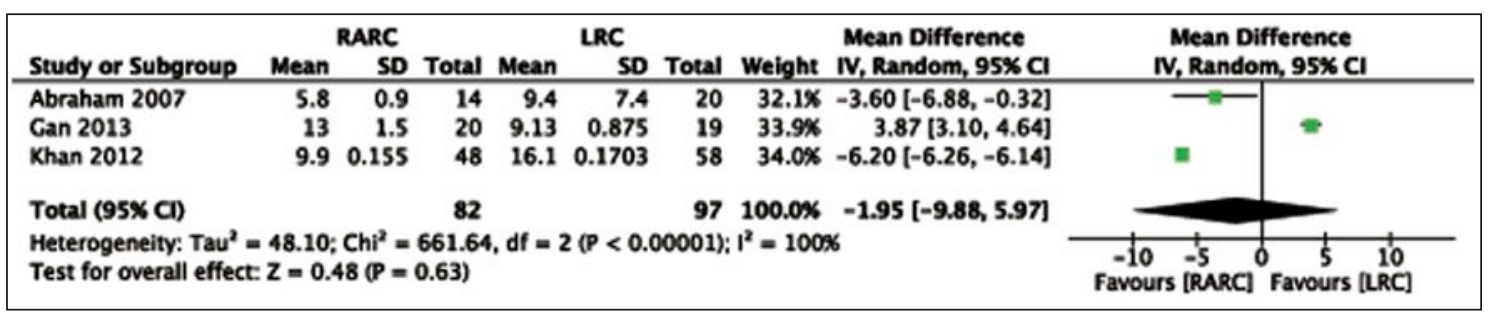

Figure 4.

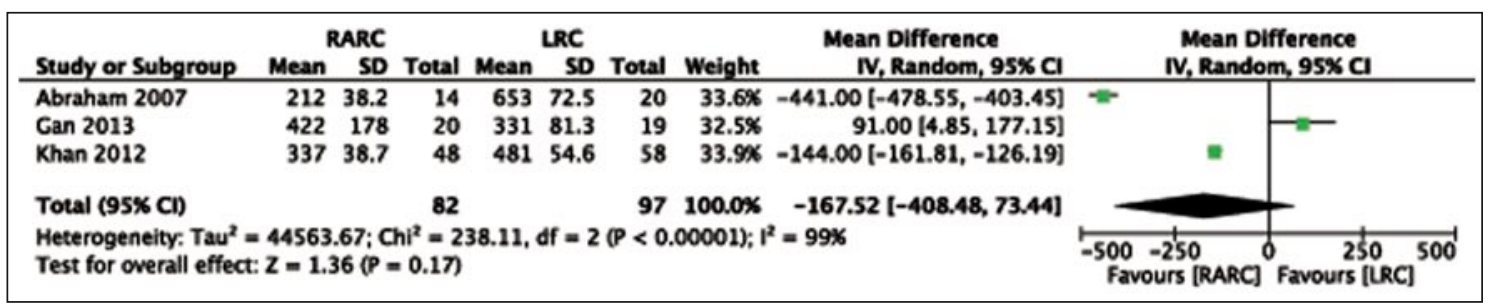

Figure 5.

\begin{tabular}{|c|c|c|c|c|c|c|c|c|c|c|}
\hline \multirow[b]{2}{*}{ Study or Subgroup } & \multirow[b]{2}{*}{ Mean } & \multicolumn{2}{|l|}{ RARC } & \multicolumn{3}{|c|}{ ORC } & \multirow[b]{2}{*}{ Weight } & \multirow{2}{*}{$\begin{array}{l}\text { Mean Difference } \\
\text { N, Random, 95\% CI }\end{array}$} & \multirow{2}{*}{\multicolumn{2}{|c|}{$\begin{array}{l}\text { Mean Difference } \\
\text { IV, Random, 95\% C }\end{array}$}} \\
\hline & & SD & Total & Mean & SD & Total & & & & \\
\hline Gan 2013 & 378 & 21.6 & 20 & 281 & 7.5 & 20 & $9.8 \%$ & $97.00[86.98,107.02]$ & & $\sim$ \\
\hline Condo 2012 & 408.5 & 1.4 & 11 & 364 & 2.66 & 15 & $10.6 x$ & $44.50[42.92,46.08]$ & & * \\
\hline Kader 2013 & 451 & 106.7 & 103 & 393 & 101.02 & 100 & $6.3 \%$ & $58.00[29.42,86.58]$ & & \\
\hline Khan 2012 & 386 & 22.9 & 48 & 320 & 27.4 & 52 & $9.8 x$ & $66.00[56.13,75.87]$ & & $\longrightarrow$ \\
\hline Knox 2013 & 468 & 0.0762 & 58 & 396 & 0.0916 & 84 & $10.6 \%$ & $72.00[71.97,72.03]$ & & • \\
\hline Musch 2014 & 410 & 68 & 100 & 351 & 92 & 42 & $5.9 \%$ & $59.00[28.15,89.85]$ & & \\
\hline Nepple 2013 & 398 & 0.0848 & 36 & 338 & 0.0762 & 29 & $10.6 \%$ & $60.00[59.96,60.04]$ & & - \\
\hline Ng 2010 & 375 & 90 & 83 & 357 & 132 & 104 & $5.7 x$ & $18.00[-13.91,49.91]$ & & \\
\hline Nix 2010 & 252 & 0.0458 & 21 & 211 & 0.0447 & 20 & $10.6 \%$ & $41.00[40.97,41.03]$ & & * \\
\hline Parekh 2013 & 301.5 & 31.5 & 20 & 283 & 20.33 & 19 & $8.6 \%$ & $18.50[1.94,35.06]$ & & \\
\hline Rhee 2006 & 638 & 46 & 7 & 507 & 110 & 23 & $2.9 \%$ & $131.00[74.59,187.41]$ & & \\
\hline Styn 2012 & 454.9 & 100 & 50 & 349.1 & 86.8 & 100 & $5.6 \%$ & $105.80[73.28,138.32]$ & & \\
\hline Sung 2012 & 578.2 & 152.9 & 35 & 500.6 & 109.7 & 104 & $3.0 \%$ & $77.60[22.73,132.47]$ & & \\
\hline Total $(95 \% \mathrm{CD})$ & & & 592 & & & 712 & $100.0 \%$ & $60.78[49.64,71.92]$ & & \\
\hline \multicolumn{9}{|c|}{$\begin{array}{l}\text { Heterogeneity: Tau }=303.39 ; \mathrm{Chi}^{2}=2428727.51 \text {, df }=12(P<0.00001) ; I^{2}=100 \% \\
\text { Test for overall effect: } Z=10.70(P<0.00001)\end{array}$} & $\begin{array}{ll}-100 & -50 \\
\text { Favours [RARC] }\end{array}$ & $\begin{array}{c}50 \\
\text { Favours [ORC] }\end{array}$ \\
\hline
\end{tabular}

complications above Clavien grade 3, including return to operating room (OR) within 30 days post-operatively and death within 90 days post-operatively. $8.51 \%$ of patients undergoing RARC had major complications with 3 deaths $(0.412 \%)$ within 90 days post-operatively. In the LRC studies, $72.1 \%$ of patients developed complications with 3 deaths $(1.087 \%)$ within 90 days post-operatively. The overall complication rate in ORC was $47.2 \%$ with an average of $8.5 \%$ of patients having major complication. There were 7 deaths $(0.64 \%)$ within 90 days post-operatively. The type of urinary diversion created with each technique was broadly classified into either conduit or bladder substitution (neo-bladder). On average $17.4 \%$ of RARC patients had a bladder substitution compared to $13.04 \%$ in LRC and $12.4 \%$ in the ORC groups and the remaining had an ileal conduit urinary diversion.

Synthesis of results

RARC versus $L R C$

As shown in Figure 2, OPT was significantly longer in RARC when compared to LRC $(\mathrm{P}=0.02$; mean weighted difference (MWD) was 47.61 with 95\% confidence interval (CI) of 8.83 to 86.40 ). There was no statistically significant difference concerning LOS $(\mathrm{P}=0.63$; MWD $=-1.95,95 \%$ $\mathrm{CI}=-9.88$ to 5.97 ) (Figure 3 ). There was also no statistical significance observed when comparing EBL $(P=0.17$; MWD $=-167.52,95 \% \mathrm{CI}=-408.48$ to 73.44 ) (Figure 4 ).

RARC versus ORC

OPT was significantly longer in RARC when compared to ORC $(\mathrm{P}=<0.00001 ; \mathrm{MWD}=60.78,95 \% \mathrm{CI}=49.64$ to 71.92) (Figure 5). Comparing other parameters of EBL, LOS and complications the analysis showed that there was significant reduction in EBL $(\mathrm{P}=<0.00001$; MWD $=-638.24,95 \% \mathrm{CI}=-850.26$ to -426.21 ) (Figure 6), LOS after RARC $(\mathrm{P}=0.004 ; \mathrm{MWD}=-1.75,95 \%$ $\mathrm{CI}=-2.94$ to -0.56 ) (Figure 7 ) and complications in the RARC group $(\mathrm{P}=<0.0001 ; \mathrm{MWD}=0.53,95 \% \mathrm{CI}=0.40$ to 0.71 ) (Figure 8). There was no statistical difference in LNY ( $\mathrm{P}=0.87$; MWD $=-0.22,95 \% \mathrm{CI}=-2.83$ to 2.39 ) (Figure 9) or PSM $(\mathrm{P}=0.42 ; \mathrm{MWD}=0.80,95 \% \mathrm{CI}=$ 0.47 to 1.37 ) (Figure 10). 
LRC versus ORC

Comparing LRC to ORC operative time was significantly

longer using LRC ( $\mathrm{P}=0.002 ; \mathrm{MWD}=34.93$, $95 \% \mathrm{CI}=$
12.76 to 57.10 ) (Figure 11). EBL was significantly reduced in the LRC group $(\mathrm{P}=0.0009$; $\mathrm{MWD}=-480.96,95 \% \mathrm{CI}=$ -765.04 to -196.88) (Figure 12). Likewise LOS was signif-

\begin{tabular}{|c|c|c|c|c|c|c|c|c|c|c|}
\hline \multirow[b]{2}{*}{ Study or Subgroup } & \multirow[b]{2}{*}{ Mean } & \multicolumn{2}{|l|}{ RARC } & \multicolumn{3}{|c|}{ ORC } & \multirow[b]{2}{*}{ Weight } & \multirow{2}{*}{$\begin{array}{l}\text { Mean Difference } \\
\text { IV, Random, } 95 \% \mathrm{Cl}\end{array}$} & \multirow{2}{*}{\multicolumn{2}{|c|}{$\begin{array}{c}\text { Mean Difference } \\
\text { IV, Random, } 95 \% \mathrm{CI}\end{array}$}} \\
\hline & & SD & Total & Mean & SD & Total & & & & \\
\hline Gan 2013 & 422 & 178 & 20 & 738 & 113 & 20 & $7.9 \%$ & $-316.00[-408.40,-223.60]$ & - & \\
\hline Gondo 2012 & 657 & 0.128 & 11 & 1,790 & 0.15 & 15 & $8.0 \%$ & $-1133.00[-1133.11,-1132.89]$ & 4 & \\
\hline Kader 2013 & 423 & 259 & 103 & 986 & 311 & 100 & $7.9 \%$ & $-563.00[-641.85,-484.15]$ & - & \\
\hline Khan 2012 & 337 & 39.2 & 48 & 1,350 & 91.3 & 52 & $8.0 \%$ & $-1013.00[-1040.18,-985.82]$ & 4 & \\
\hline Knox 2013 & 276 & 48.5 & 58 & 1,522 & 369.1 & 84 & $7.9 \%$ & $-1246.00[-1325.91,-1166.09]$ & 4 & \\
\hline Musch 2014 & 351 & 170 & 100 & 810 & 621 & 42 & $7.5 \%$ & $-459.00[-649.74,-268.26]$ & & \\
\hline Nepple 2013 & 675 & 0.0848 & 36 & 1,497 & 0.0762 & 29 & $8.0 \%$ & $-822.00[-822.04,-821.96]$ & - & \\
\hline $\mathrm{Ng} 2010$ & 460 & 299 & 83 & 1,172 & 916 & 104 & $7.5 \%$ & $-712.00[-899.43,-524.57]$ & & \\
\hline Nix 2010 & 258 & 0.0458 & 21 & 575 & 0.0447 & 20 & $8.0 \%$ & $-317.00[-317.03,-316.97]$ & - & \\
\hline Parekh 2013 & 466 & 116 & 20 & 781 & 181 & 19 & $7.9 \%$ & $-315.00[-410.96,-219.04]$ & $\longrightarrow$ & \\
\hline Rhee 2006 & 479 & 551 & 7 & 1,109 & 398 & 23 & $6.0 \%$ & $-630.00[-1069.39,-190.61]$ & & \\
\hline Styn 2012 & 350 & 375 & 50 & 475 & 483 & 100 & $7.7 \%$ & $-125.00[-265.59,15.59]$ & & \\
\hline Sung 2012 & 448 & 231 & 35 & 1,063 & 893 & 104 & $7.5 \%$ & $-615.00[-802.92,-427.08]$ & & \\
\hline Total $(95 \% \mathrm{Cl})$ & & & 592 & & & 712 & $100.0 \%$ & $-638.24[-850.26,-426.21]$ & & \\
\hline $\begin{array}{l}\text { Heterogeneity: } \mathrm{Tau}^{2} \\
\text { Test for overall effect }\end{array}$ & $\begin{array}{l}1459 \\
Z=5\end{array}$ & $\begin{array}{l}\text { 7.49; } \mathrm{Chi}^{2} \\
90(\mathrm{P}<0 .\end{array}$ & $\begin{array}{l}i^{2}=559 \\
.00001)\end{array}$ & 958524 & $0.84, \mathrm{df}=$ & $=12(P$ & $<0.0000$ & $01) ;\left.\right|^{2}=100 \%$ & $\begin{array}{c}1000-500 \\
\text { Favours [RARC] }\end{array}$ & $\begin{array}{|cc|}500 & 1000 \\
\text { Favours [ORC] }\end{array}$ \\
\hline
\end{tabular}

\begin{tabular}{|c|c|c|c|c|c|c|c|c|c|c|}
\hline \multirow[b]{2}{*}{ Study or Subgroup } & \multicolumn{3}{|c|}{ RARC } & \multicolumn{3}{|c|}{ ORC } & \multirow[b]{2}{*}{ Weight } & \multirow{2}{*}{$\begin{array}{l}\text { Mean Difference } \\
\text { NV, Random, 95\% CI }\end{array}$} & \multirow{2}{*}{\multicolumn{2}{|c|}{$\begin{array}{c}\text { Mean Difference } \\
\text { IV, Random, 95\% CI }\end{array}$}} \\
\hline & Mean & SD & Total & Mean & SD & Total & & & & \\
\hline Gan 2013 & 11.3 & 2.25 & 20 & 13 & 1.5 & 20 & $9.3 \%$ & $-1.70[-2.89,-0.51]$ & - & \\
\hline Condo 2012 & 40.2 & 1.63 & 11 & 37 & 1.908 & 15 & $9.1 \%$ & $3.20[1.84,4.56]$ & & $\sim$ \\
\hline Kader 2013 & 6 & 0.5 & 103 & 8 & 0.833 & 100 & $10.3 \%$ & $-2.00[-2.19,-1.81]$ & - & \\
\hline Khan 2012 & 9.9 & 5.42 & 48 & 19.2 & 9.43 & 52 & $6.2 \%$ & $-9.30[-12.29,-6.31]$ & & \\
\hline Knox 2013 & 6.3 & 0.45 & 58 & 10.8 & 1.24 & 84 & $10.2 \%$ & $-4.50[-4.79,-4.21]$ & * & \\
\hline Musch 2014 & 17.1 & 7.6 & 100 & 19.9 & 12 & 42 & $4.9 \%$ & $-2.80[-6.72,1.12]$ & & \\
\hline Nepple 2013 & 7.9 & 2.2 & 36 & 9.6 & 1.97 & 29 & $9.6 \%$ & $-1.70[-2.72,-0.68]$ & - & \\
\hline Ng 2010 & 5.5 & 4.17 & 83 & 8 & 9.5 & 104 & $7.9 \%$ & $-2.50[-4.53,-0.47]$ & & \\
\hline Nix 2010 & 5.1 & 1.95 & 21 & 6 & 1.906 & 20 & $9.3 \%$ & $-0.90[-2.08,0.28]$ & & \\
\hline Parekh 2013 & 6.63 & 1.13 & 20 & 6.83 & 0.825 & 19 & $10.0 \%$ & $-0.20[-0.82,0.42]$ & & \\
\hline Rhee 2006 & 11 & 5 & 7 & 13 & 11 & 23 & $3.0 \%$ & $-2.00[-7.82,3.82]$ & & \\
\hline Styn 2012 & 9.5 & 8.8 & 50 & 10.2 & 8.4 & 100 & $6.3 \%$ & $-0.70[-3.64,2.24]$ & & \\
\hline Sung 2012 & 28.9 & 11.9 & 35 & 27.1 & 13.4 & 104 & $3.9 \%$ & $1.80[-2.91,6.51]$ & & \\
\hline Total $(95 \% \mathrm{CD})$ & & & 592 & & & 712 & $100.0 \%$ & $-1.75[-2.94,-0.56]$ & & \\
\hline \multicolumn{9}{|c|}{$\begin{array}{l}\text { Heterogeneity: } \text { Tau }^{2}=3.58 ; \mathrm{Chi}^{2}=366.06, \mathrm{df}=12(\mathrm{P}<0.00001) ; \mathrm{I}^{2}=97 \% \\
\text { Test for overall effect: } \mathrm{Z}=2.88(\mathrm{P}=0.004)\end{array}$} & $\begin{array}{lll}-10 & -5 & 0 \\
\text { Favours [RARC] }\end{array}$ & $\begin{array}{cc}5 & 10 \\
\text { Favours [ORC] }\end{array}$ \\
\hline
\end{tabular}

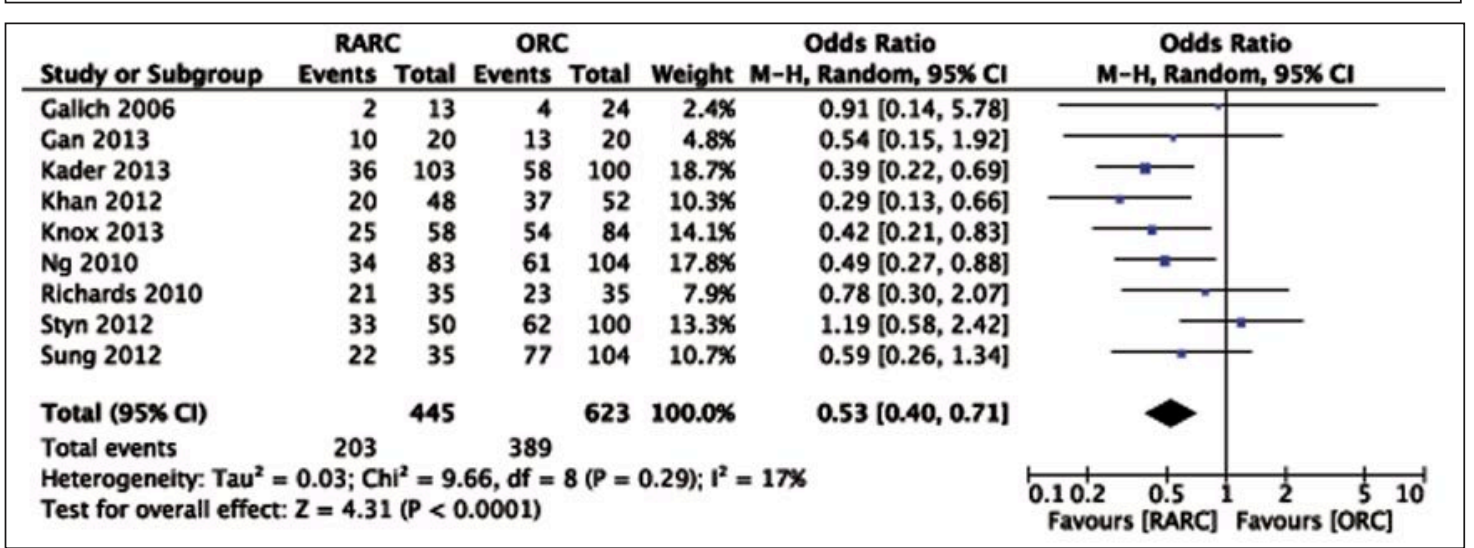

\begin{tabular}{|c|c|c|c|c|c|c|c|c|c|c|}
\hline \multirow[b]{2}{*}{ Study or Subgroup } & \multicolumn{3}{|c|}{ RARC } & \multicolumn{3}{|c|}{ ORC } & \multirow[b]{2}{*}{ Weight } & \multirow{2}{*}{$\begin{array}{l}\text { Mean Difference } \\
\text { IV, Random, } 95 \% \text { CI }\end{array}$} & \multirow{2}{*}{\multicolumn{2}{|c|}{$\begin{array}{c}\text { Mean Difference } \\
\text { IV, Random, 95\% C }\end{array}$}} \\
\hline & Mean & SD & Total & Mean & SD & Total & & & & \\
\hline Abaza 2012 & 36.9 & 14.8 & 35 & 37.5 & 13.2 & 120 & 8.18 & $-0.60[-6.04,4.84]$ & & \\
\hline Can 2013 & 16.25 & 8.02 & 20 & 18.8 & 7.59 & 20 & $8.7 \%$ & $-2.55[-7.39,2.29]$ & & \\
\hline Condo 2012 & 13.8 & 6.4 & 11 & 20.7 & 7.5 & 15 & $8.2 \%$ & $-6.90[-12.26,-1.54]$ & & \\
\hline Khan 2012 & 16 & 5.5 & 48 & 11 & 7.25 & 52 & 11.28 & $5.00[2.49,7.51]$ & & \\
\hline Musch 2014 & 27.5 & 11 & 100 & 19.6 & 8.8 & 42 & $10.3 \%$ & $7.90[4.47,11.33]$ & & \\
\hline Nepple 2013 & 14 & 7.3 & 36 & 17 & 5.8 & 29 & $10.5 \%$ & $-3.00[-6.18,0.18]$ & & \\
\hline $\mathrm{Ng} 2010$ & 15.7 & 13.2 & 83 & 17.9 & 10.4 & 104 & $10.2 \%$ & $-2.20[-5.67,1.27]$ & & \\
\hline Nix 2010 & 18.5 & 5.5 & 21 & 18.3 & 2.8 & 20 & $11.1 \%$ & $0.20[-2.45,2.85]$ & & \\
\hline Pruthi 2007 & 16 & 4.5 & 20 & 19 & 4.5 & 24 & 11.18 & $-3.00[-5.67,-0.33]$ & & \\
\hline Styn 2012 & 15.2 & 9.5 & 50 & 14.3 & 9.1 & 100 & $10.5 \%$ & $0.90[-2.28,4.08]$ & & \\
\hline Total $(95 \% \mathrm{CD})$ & & & 424 & & & 526 & $100.0 \%$ & $-0.22[-2.83,2.39]$ & & \\
\hline \multicolumn{9}{|c|}{$\begin{array}{l}\text { Heterogeneity: Tau }{ }^{2}=14.17 ; \mathrm{Chi}^{2}=52.87, \mathrm{df}=9(\mathrm{P}<0.00001) ; \mathrm{I}^{2}=83 \% \\
\text { Test for overall effect: } \mathrm{Z}=0.16(\mathrm{P}=0.87)\end{array}$} & $\stackrel{-20-10}{\text { Favours [RARC] }}$ & ${ }_{\text {Favours [ORC] }}^{10} 20$ \\
\hline
\end{tabular}

Figure 7.

Figure 8.

Figure 9.

Figure 6.

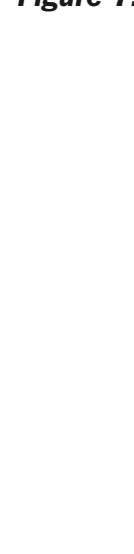

Fine 
whether complication rate is significantly reduced when comparing RARC to LRC.

RARC takes longer to do than ORC but produces better surgical outcomes with reduced EBL, shorter LOS and fewer complications. There are equivalent oncological outcomes (LNY and PSM.)

When comparing LRC to ORC, LNY is equivalent but there is an increase in OPT, reduction in EBL and shorter LOS using the laparoscopic approach. The results therefore are in favor of using LRC in preference to ORC. It may be that it is when the robotic technique is used with intracorporeal urinary diversion, as opposed to extracorporeal, that it is superior to LRC. Most studies included used extracorporeal urinary diversion.

Intracorporeal urinary diversion has been demonstrated to be technically feasible with good oncological outcomes $(13,30)$. More data is needed to assess long-term outcomes.

The difficulty in obtaining data on complications results from a lack of consistency in reporting complications. In this paper the Clavien-Dindo system was used and it is broadly accepted as the better current standard for reporting of surgical complications. All future trials assessing the complications in radical cystectomy should use this system to facilitate universal comparison (31).

One outcome in which data was lacking was PSM. A study using data from the International Robotic Cystectomy Consortium (IRCC) (32) found that the rate of PSM was similar between RARC and ORC, consistent with the results of this meta-analysis. Variables associated with increased probability of PSM using RARC included older age, higher pathological $\mathrm{T}$ stage and lymph node positivity.

A similar study by the IRCC reviewed the outcomes of extended lymph node dissection, an essential part of radical cystectomy (33). Similar lymph node yields were obtained in RARC and ORC, which were found to be the case in this study. The study also identified that high volume institutions ( $\geq 100$ cases) had 3.46-times increased probability of carrying out extended lymph node dissection (LND).

It is crucial that survival data is reported in the studies of different surgical techniques to see if technology is having an influence on the survival of these patients.

This would only be possible with longer follow up after surgical procedures. Kaplan-Meier plots to compare survival rates between the three operative techniques would prove valuable in assessing the evidence for RARC.

Guru et al. (34) have shown that surgical and oncological outcomes constantly improve with each RARC case the surgeon performs. The learning curve for RARC was defined by results from the IRCC. Using proxy measurements for RARC quality such as OPT, EBL, LNY and margin positivity it was found that acceptable proficiency in the procedure was attained by the $30^{\text {th }}$ case.

The cost of RARC is estimated to be about $\$ 20,000$ per case and is an important factor to consider when evaluating the use of RARC. Lee et al. (35) have found that RARC is less expensive than ORC when ileal conduit or continent cutaneous diversion is performed. The main driver of cost was LOS and though material cost was higher with RARC, in high-volume centres RARC can be more cost- effective particularly with ileal conduit urinary diversion. The true benefit of RARC may lie in the improved ergonomics of the robotic system. The more comfortable operating system may cause less fatigue to the surgeon as compared to laparoscopic methods, thereby leading to fewer errors. This was shown by Elhage et al. (36) where time taken to perform a suturing task was not only shorter compared to laparoscopic and open, but there were also fewer errors made when compared to the laparoscopic method. The major limitation of this study is the possibility of bias. When evaluating surgical procedures there is always a lack of blinding and natural variation in both the skill of the surgeon pathology of different cases. This is reflected in the significant heterogeneity found in the forest plots of this study. Inclusion criteria varied among studies as well as there being different systems of followup with differing outcome definitions. Publication bias is also a possibility that was not factored into this study.

\section{CONCLUSIONS}

The results of this meta-analysis shows that LRC provides better outcomes than ORC but that RARC provides similar outcomes to LRC, only with longer OPT. More randomised control trials are required to provide conclusive evidence to show whether or not RARC is in fact a better alternative to ORC or LRC. These studies must use a unified system for the classification of complications and assess both surgical and oncological outcomes. More data is also needed on the ergonomics, learning curve, cost-effectiveness and patient-perspectives of RARC.

\section{ACKNOWLEDGEMENTS}

P.D. acknowledges financial support from the National Institute for Health Research (NIHR) Biomedical Research Centre based at Guy's and St Thomas' NHS Foundation Trust and King's College London. The views expressed are those of the author(s) and not necessarily those of the NHS, the NIHR or the Department of Health. P.D. also acknowledges the support of the MRC Centre for Transplantation, London Deanery, London School of Surgery and Olympus. P.D., S.K. and K.A. acknowledge funding for the SIMULATE project from the Urology Foundation (TUF) and the BAUS. KA and PD acknowledge educational funding from the Royal College of Surgeons of England.

\section{REFERENCES}

1. Springer C, Mohammed N, Alba S, et al. Laparoscopic radical cystectomy with extracorporeal ileal neobladder for muscle-invasive urothelial carcinoma of the bladder: technique and short-term outcomes. World J Urol. 2013; 1-6.

2. Khan MS, Elhage O, Challacombe B, et al. Analysis of Early Complications of Robotic-assisted Radical Cystectomy Using a Standardized Reporting System. Urology. 2011; 2:357-362.

3. Dindo D, Demartines N, Clavien P. Classification of surgical complications: a new proposal with evaluation in a cohort of 6336 patients and results of a survey. Ann Surg. 2004; 240:205-213.

4. Wong WC, Cheung CS, Hart GJ. Development of a quality assess- 
ment tool for systematic reviews of observational studies (QATSO) of HIV prevalence in men having sex with men and associated risk behaviours. Emerg Themes Epidemiol. 2008; 5:23.

5. Moher D, Cook DJ, Eastwood S, et al. Improving the quality of reports of meta-analyses of randomised controlled trials: the QUOROM statement. The Lancet. 1999; 354:1896-1900.

6. Abaza R, Dangle PP, Gong MC, et al. Quality of lymphadenectomy is equivalent with robotic and open cystectomy using an extended template. J Urol. 2012; 187:1200-1204.

7. Abraham JBA, Young JL, Box GN, et al. Comparative analysis of laparoscopic and robot-assisted radical cystectomy with ileal conduit urinary diversion. J Endourol. 2007; 21:1473-1480.

8. Galich A, Sterrett S, Nazemi T, et al. Comparative analysis of early perioperative outcomes following radical cystectomy by either the robotic or open method. JSLS, JSLS. 2006; 10:145-150.

9. Gondo T, Yoshioka K, Nakagami Y, et al. Robotic versus open radical cystectomy: Prospective comparison of perioperative and pathologic outcomes in japan. Jpn J Clin Oncol. 2012; 42:625-631.

10. Kader AK, Richards KA, Krane LS, et al. Robot-assisted laparoscopic vs open radical cystectomy: comparison of complications and perioperative oncological outcomes in 200 patients. BJU Int. 2013; 112:E290-E294.

11. Khan MS, Challacombe B, Elhage O, et al. A dual-centre, cohort comparison of open, laparoscopic and robotic-assisted radical cystectomy. Int J Clin Pract. 2012; 66:656-662.

12. Knox ML, El-Galley R, Busby JE. Robotic versus open radical cystectomy: identification of patients who benefit from the robotic approach. J Endourol. 2013; 27:40-44.

13. Jonsson MN, Adding LC, Hosseini A, et al. Robot-assisted radical cystectomy with intracorporeal urinary diversion in patients with transitional cell carcinoma of the bladder. Eur Urol. 2011; 60:1066-1073.

14. Nepple KG, Strope SA, Grubb III RL, et al. Early oncologic outcomes of robotic vs. open radical cystectomy for urothelial cancer. Urol Oncol, 2013; 31:894-898.

15. Ng CK, Kauffman EC, Lee M, et al. A Comparison of Postoperative Complications in Open versus Robotic Cystectomy. Eur Urol. 2010; 57:274-282.

16. Nix J, Smith A, Kurpad R, et al. Prospective randomized controlled trial of robotic versus open radical cystectomy for bladder cancer: perioperative and pathologic results. Eur Urol. 2010; 57:196-201.

17. Parekh DJ, Messer J, Fitzgerald J, et al. Perioperative outcomes and oncologic efficacy from a pilot prospective randomized clinical trial of open versus robotic assisted radical cystectomy. J Urol. 2013; 189:474-479.

18. Porpiglia F, Renard J, Billia M, et al. Open versus laparoscopyassisted radical cystectomy: Results of a prospective study. J Endourol. 2007; 21:325-329.

19. Pruthi RS, Wallen EM. Robotic assisted laparoscopic radical cystoprostatectomy: operative and pathological outcomes. J Urol. 2007; 178:814-818.

20. Richards KA, Hemal AK, Kader AK, et al. Robot assisted laparoscopic pelvic lymphadenectomy at the time of radical cystectomy rivals that of open surgery: single institution report. Urology. 2010; 76:1400-1404.

21. Styn NR, Montgomery JS, Wood DP, et al. Matched comparison of robotic-assisted and open radical cystectomy. Urology. 2012; 79:1303-1308.

22. Sung HH, Ahn J, Seo SI, et al. A comparison of early complications between open and robot-assisted radical cystectomy. J Endourol. 2012; 26:670-675.
23. Gan C, Ismail F, Cheung G, et al. 1033 A pilot prospective single-centre 3-arm randomised controlled trial of open, robotic and laparoscopic (CORAL) radical cystectomy for bladder cancer. Eur Urol Suppl. 2013; 12:e1033-e1034.

24. Ha U, Kim SI, Kim SJ, et al. Laparoscopic versus open radical cystectomy for the management of bladder cancer: Mid-term oncological outcome. Int J Urol. 2010; 17:55-61.

25. Guillotreau J, Gamé X, Mouzin M, et al. Radical cystectomy for bladder cancer: morbidity of laparoscopic versus open surgery. J Urol. 2009; 181:554-9.

26. Haber G-P, Crouzet S, Gill IS. Laparoscopic and robotic assisted radical cystectomy for bladder cancer: a critical analysis. Eur Urol. 2008; 54:54-64.

27. Lin T, Fan $X$, Zhang $C$, et al. A prospective randomised controlled trial of laparoscopic vs open radical cystectomy for bladder cancer: perioperative and oncologic outcomes with 5-year follow-up. Br J Cancer. 2014; 110:842-9.

28. Musch M, Janowski M, Steves A, et al. Comparison of early postoperative morbidity after robot-assisted and open radical cystectomy: results of a prospective observational study. BJU international. 2014; 113:458-67.

29. Rhee JJ, Lebeau S, Smolkin M, Theodorescu D. Radical cystectomy with ileal conduit diversion: early prospective evaluation of the impact of robotic assistance. BJU International. 2006; 98:1059-63.

30. Sala LG, Matsunaga GS, Corica FA, et al. Robot-assisted laparoscopic radical cystoprostatectomy and totally intracorporeal ileal neobladder. J Endourol. 2006; 20:233-236.

31. Johar RS, Hayn MH, Stegemann AP, et al. Complications after robot-assisted radical cystectomy: results from the International Robotic Cystectomy Consortium. Eur Urol. 2013; 64:52-57.

32. Hellenthal N, Hussain A, Andrews P, et al. Status of surgical margins after robot-assisted radical cystectomy: RESULTS from the International Robotic cystectomy consortium. J Urol 2010; 183:e636-e637.

33. Marshall SJ, Hayn MH, Stegemann AP, et al. Impact of surgeon and volume on extended lymphadenectomy at the time of robotassisted radical cystectomy: Results from the International Robotic Cystectomy Consortium (IRCC). BJU Int. 2013; 111:1075-1080.

34. Guru KA, Perlmutter AE, Butt ZM, et al. The learning curve for robot-assisted radical cystectomy. JSLS. 2009; 13:509-514.

35. Lee $\mathrm{R}, \mathrm{Ng} \mathrm{CK}$, Shariat SF, et al. The economics of robotic cystectomy: Cost comparison of open versus robotic cystectomy. BJU Int. 2011; 108:1886-1892.

36. Elhage O, Challacombe B, Shortland A, et al. An assessment of the physical impact of complex surgical tasks on surgeon errors and discomfort: a comparison between robotic-assisted, laparoscopic and open approaches. BJU Int 2014. doi: 10.1111/bju.12680.

\section{Correspondence}

Thomas Fonseka (BSC)

King's College London School of Medicine, London, UK

Kamran Ahmed, MRCS, PhD (Correspondent Author)

kamran.ahmed@kcl.ac.uk

Saied Froghi, MBBS

Prokar Dasgupta, MSC, MD, FEBU, FRCS Urol

Mohammad Shamim Khan, OBE, FEBU, FRCS Urol

NIHR Academic Clinical Lecturer /Specialist Registrar in Urology

MRC Centre for Transplantation, Guys Hospital

St Thomas Street, London SEl 9RT, UK

Shahid A Khan, FRCS Urol

Surrey and Sussex Healthcare NHS Hospital, Redhill, UK 